\title{
Sebaceous Gland of the Eyelash
}

National Cancer Institute

\section{Source}

National Cancer Institute. Sebaceous Gland of the Eyelash. NCI Thesaurus. Code C33521.

The small glands located within the epidermis of the eyelid that produce and secrete an oily substance that lubricates the eyelashes. 